\title{
Evaluation of non-surgical therapy on glutathione levels in chronic periodontitis
}

\author{
Pooja Palwankar ${ }^{1}$, Minakshi Rana $^{1}$, Kapil Arora $^{2}$, C. Deepthy ${ }^{3}$
}

Correspondence: Dr. Pooja Palwankar Email: poojapalwankar@gmail.com

\author{
'Department of Periodontology, Manav Rachna Dental \\ College, Faridabad, Haryana, India, \\ ${ }^{2}$ Department of Public Health Dentistry, Manav Rachna \\ Dental College, Faridabad, Haryana, India, \\ ${ }^{3}$ Department of Biochemistry, Manav Rachna Dental \\ College, Faridabad, Haryana, India
}

\section{ABSTRACT}

Objective: To compare the levels of glutathione (GSH), both oxidized and reduced forms in patients with and without chronic periodontitis in gingival crevicular fluid (GCF). Materials and Methods: Twenty GCF samples from maxillary quadrants were collected using capillary micropipettes from the chronic periodontitis patients (test group) at baseline before treatment, at 1-month, 3 months, and 6 months after scaling and root planing and samples from 20 patients without chronic periodontitis (control group) from maxillary quadrants were also collected. GSH, oxidized glutathione (GSSG) levels and GSH: GSSG ratios were determined using the spectrophotometric method. Statistical Analysis: Results were concluded for the test over control groups using paired Student's $t$-test. Results: Lower concentrations of GSH $(P<0.001)$ and GSSG $(P<0.001)$ were detected in GCF in patients with chronic periodontitis (test group) than patients without chronic periodontitis (control group) at baseline. Treatment had a significant effect in improving the GSH and reducing GSSG levels postscaling and root planing at 1-month and 3 months but not significant effect at 6 months. Scaling and root planing increased the GSH: GSSG ratio $(P<0.001)$ in the test group as compared to the control group $(P<0.001)$. Conclusions: The concentrations of GSH within GCF are reduced in chronic periodontitis patients. Scaling and root planing (nonsurgical therapy) restores GSH concentration in GCF post 1-month and 3 months along with redox balance (GSH: GSSG ratio), but at 6 months the balance is not maintained. Adjunctive use of micronutritional supplements to boost antioxidant concentration in tissues by preserving GSH or by elevating its level at the inflamed sites is recommended, as nonsurgical periodontal therapy alone is not able to maintain redox balance for longer duration.

Key words: Chronic periodontitis, oxidized glutathione, redox balance, reduced glutathione

\section{INTRODUCTION}

Periodontitis is a chronic inflammatory disease of supporting tissues of the teeth in response to noxious stimuli whether mechanical, chemical or infectious, resulting in progressive destruction of periodontal apparatus, hence leading to pocket formation, recession or both. ${ }^{[1-5]}$ The tissue destruction in periodontitis has been attributed to the production of enzymes and reactive oxygen species (ROS) by polymorphonuclear cells and other cells. It has been demonstrated that patients with periodontitis have higher oxidative DNA and lipid damage biomarkers and lower antioxidant (AO) enzymatic activities in saliva than healthy subjects. ${ }^{[6]}$ There are a variety of defense mechanisms in the body to combat excessive ROS production, and AOs are one among these. AOs are those substances when present at a low concentration compared to those of an oxidizable substrate significantly delays or prevents oxidation of the substrate. ${ }^{[7]}$ They act at three different levels - prevention, interception, and repair. ${ }^{[8]}$ Superoxide and hydrogen peroxide are the main oxidative species produced, which are either

This is an open access article distributed under the terms of the Creative Commons Attribution-NonCommercial-ShareAlike 3.0 License, which allows others to remix, tweak, and build upon the work non-commercially, as long as the author is credited and the new creations are licensed under the identical terms.

For reprints contact: reprints@medknow.com

How to cite this article: Palwankar P, Rana M, Arora K, Deepthy C.
Evaluation of non-surgical therapy on glutathione levels in chronic
periodontitis. Eur J Dent $2015 ; 9: 415-22$.
DOI: $10.4103 / 1305-7456.163226$


enzymatically eliminated by preventive AOs, or metal ions are sequestrated, hence preventing Fenton reactions and subsequent hydroxyl radical formation ${ }^{[9]}$ e.g. - catalase, glutathione (GSH) peroxidase, 7S-transferase. Interception involves scavenging/chain breaking AOs which inhibit chain initiation, chain propagation and also lipid peroxidation e.g. - lipophilic substances such as ubiquinol, Vitamin A, Vitamin E, carotenoids and hydrophilic substances like uric acid, ascorbic acid, albumin, and bilirubin. At repair level various AOs function by repairing the damaged and reconstituting membranes. These include DNA repair enzymes, protease, transferase, and lipase.

When the level of ROS increases intracellularly, the cellular AO defenses are insufficient to maintain these harmful molecules, this condition is generally referred to as "oxidative stress." The concept of "oxidative stress" dates back to 1986 and was elaborated as the relation between free radicals and disease ${ }^{[10]} \mathrm{GSH}$ is a ubiquitous tripeptide made from the combination of three amino acids, that is, cysteine, glutamate, and glycine. It is a low molecular weight thiol (up to 5-10 $\mathrm{mM}$ ) present in the cell and existing in two forms which are oxidized glutathione (GSSG) and reduced GSH forms. ${ }^{[11]}$ Out of the total GSH present in the body, the reduced GSH constitutes about $90 \%$, and GSSG is $10 \%$. For survival of cell, it is imperative to maintain optimal GSH: GSSG ratios. Oxidative damage results when the there is a deficiency of reduced GSH in the cell, which puts it at risk for oxidative damage. ${ }^{[11]}$ Reduced GSH plays three major functions in the body. ${ }^{[12]}$

- Anti-oxidant function [Figure 1]

- Detoxification function

- Immune function.

The purpose of this study is to compare the levels of GSH, both oxidized and reduced forms in patients with and without chronic periodontitis in gingival crevicular fluid (GCF).

\section{MATERIALS AND METHODS}

\section{Study groups and design}

This study was a 6 months randomized case-control study, which was conducted at the Department of Periodontology. An approval for the study was obtained from the ethical committee, and written informed consent was obtained before enrolling the subjects for the study. The study enrolled 40 patients, which included patients with chronic periodontitis $n=20$ (test group) and patients

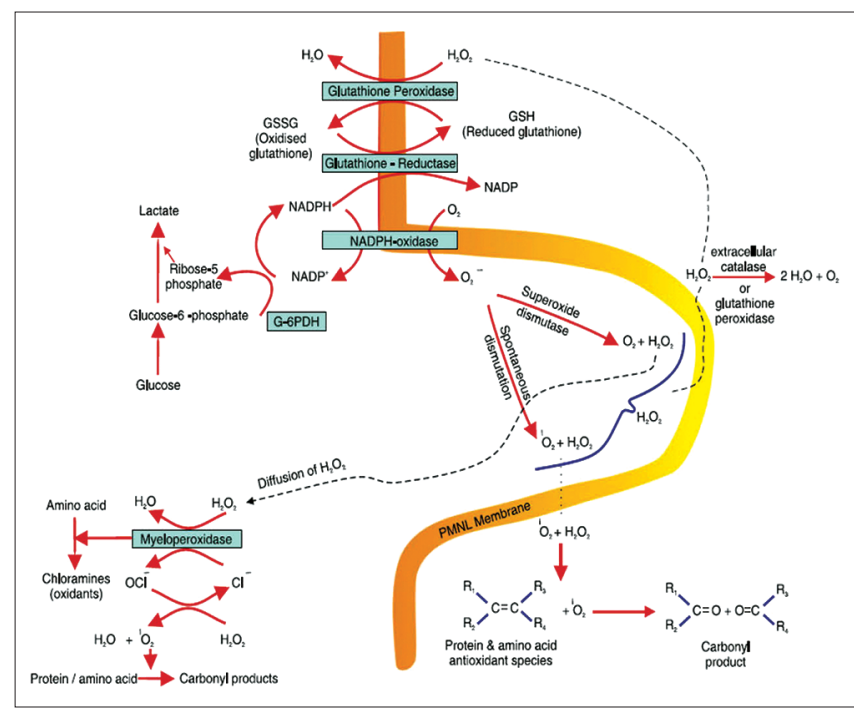

Figure 1: Schematic representation of the biochemical interactions between neutrophil superoxide and glutathione

without chronic periodontitis $n=20$ (control group). Subjects with no relevant medical history of tobacco usage were enrolled for the study. Subjects in the test group were selected on the basis of clinical criteria of having at least two nonadjacent sites per quadrant with probing pocket depths $\geq 5 \mathrm{~mm}$, along with bleeding on probing and demonstrable radiographic bone loss. Control patients did not show evidence of attachment loss or probing pocket depths $\geq 3 \mathrm{~mm}$ and bleeding scores were below $10 \%$. The subjects with a history of taking vitamin supplements, anti-inflammatory or antibiotic medication in the preceding 3 months and pregnant ladies and patient with special dietary needs were not included in the study. The study was started in January 2013 and ended in July 2014. After enrollment, baseline GCF samples were collected, before recording clinical measures which included Silness and Loe plaque index, Loe and Silness gingival index, Muhlemann and Son sulcus bleeding index and probing pocket depth. After data collection, chronic periodontitis patients (test group) underwent scaling and root planing and oral hygiene instruction which included brushing technique, dental flossing were reinforced at baseline. Patient without chronic periodontitis (control group) received only oral hygiene instructions. Subjects were then recalled at 1-month, 3 months and 6 months posttherapy for recording clinical and biochemical parameters.

\section{Gingival crevicular fluid collection and glutathione estimation}

GCF samples were collected from mesiobuccal/ distolingual sites on any teeth in the maxillary quadrant (test and control group) using microcapillary pipettes. GCF samples were then 
immediately transferred to top sealed cuvettes to prevent oxidation of AOs present in it. Levels of reduced and GSSG were analyzed using "beutler" spectrophotometric test. ${ }^{[13]}$

\section{Data analysis}

All the results of this study were obtained by comparing intragroup and intergroup parameters, at various designated phases at baseline, $1^{\text {st }}$ month, $3^{\text {rd }}$ month and $6^{\text {th }}$ month for test over control groups using paired Student's $t$-test using software Computer software SPSS version 20 (IBM SPSS Inc, Chicago, IL, USA).

\section{RESULTS}

\section{Clinical data}

The nonsurgical periodontal therapy provided to the test group resulted in observed reductions of whole mouth mean plaque scores $(P<0.001)$, mean gingival scores, and mean sulcus bleeding index scores in the group $(P<0.001)$ Which in turn showed reduced inflammation as compared to control group [Tables 1-6].
Glutathione levels in gingival crevicular fluid

In all groups, mean reduced GSH and GSSG levels were detected in the millimolar range (range: 0.30-5.14 mM [Table 7]). Lower levels of GSH and GSSG concentrations were reported in the GCF from chronic periodontitis patients before and after treatment, compared with those detected in nonchronic periodontitis subjects (control group) [Table 8].

Comparative analysis of difference in mean GSH and GSSG value for test group was done at different time intervals, namely, baseline - 1-month, baseline - 3 months, baseline - 6 months, 1-3 months and 1-6 months. The differences were observed to be statistically significant $(P<0.001)$ at a confidence interval of $95 \%$, but statistically nonsignificant at the 3-6 months interval [Tables 9 and 10]. Comparison of mean GSH and GSSG score with a standard deviation between test and control group is depicted [Figures 2 and 3] GSH and GSSG value for control group were depicted at different time intervals, namely, baseline - 1 month, baseline - 3

\begin{tabular}{|c|c|c|c|c|c|c|c|c|}
\hline Time interval & Number of subjects & Mean & SD & Mean & SD & $t$ & $P$ & Inference \\
\hline Baseline- $1^{\text {st }}$ month & 22 & 1.98 & 0.23 & 0.92 & 0.50 & 9.178 & $<0.001$ & Statistically significant \\
\hline Baseline- $3^{\text {rd }}$ month & 21 & 2.00 & 0.21 & 0.84 & 0.53 & 9.483 & $<0.001$ & Statistically significant \\
\hline Baseline- $6^{\text {th }}$ month & 21 & 2.00 & 0.21 & 0.86 & 0.49 & 9.635 & $<0.001$ & Statistically significant \\
\hline $1^{\text {st }}$ month- $3^{\text {rd }}$ month & 21 & 0.90 & 0.51 & 0.84 & 0.53 & 0.835 & 0.414 & Statistically nonsignificant \\
\hline $1^{\text {st }}$ month- $6^{\text {th }}$ month & 21 & 0.90 & 0.51 & 0.86 & 0.49 & 0.393 & 0.699 & Statistically nonsignificant \\
\hline $3^{\text {rd }}$ month- $6^{\text {th }}$ month & 21 & 0.84 & 0.53 & 0.86 & 0.49 & 0.419 & 0.68 & Statistically nonsignificant \\
\hline
\end{tabular}

\begin{tabular}{|c|c|c|c|c|c|c|c|c|}
\hline Time interval & Number of subjects & Mean & SD & Mean & SD & $t$ & $\boldsymbol{P}$ & Inference \\
\hline Baseline- $1^{\text {st }}$ month & 22 & 2.23 & 1.31 & 0.86 & 0.49 & 4.25 & $<0.001$ & Statistically significant \\
\hline Baseline- $3^{\text {rd }}$ month & 21 & 2.25 & 1.34 & 0.87 & 0.56 & 4.042 & 0.001 & Statistically nonsignificant \\
\hline Baseline- $6^{\text {th }}$ month & 21 & 2.25 & 1.34 & 1.00 & 0.67 & 3.471 & 0.002 & Statistically nonsignificant \\
\hline $1^{\text {st }}$ month- $3^{\text {rd }}$ month & 21 & 0.84 & 0.49 & 0.87 & 0.56 & 0.528 & 0.603 & Statistically nonsignificant \\
\hline $1^{\text {st }}$ month- $6^{\text {th }}$ month & 21 & 0.84 & 0.49 & 1.00 & 0.67 & 1.778 & 0.091 & Statistically nonsignificant \\
\hline $3^{\text {rd }}$ month- $6^{\text {th }}$ month & 21 & 0.87 & 0.56 & 1.00 & 0.67 & 1.961 & 0.064 & Statistically nonsignificant \\
\hline
\end{tabular}

SD: Standard deviation

\begin{tabular}{|c|c|c|c|c|c|c|c|c|}
\hline Time interval & Number of subjects & Mean & SD & Mean & SD & $t$ & $P$ & Inference \\
\hline Baseline- $1^{\text {st }}$ month & 22 & 2.20 & 0.79 & 0.93 & 0.48 & 7.341 & $<0.001$ & Statistically significant \\
\hline Baseline- $3^{\text {rd }}$ month & 21 & 2.21 & 0.81 & 0.94 & 0.63 & 4.968 & $<0.001$ & Statistically significant \\
\hline Baseline- $6^{\text {th }}$ month & 21 & 2.21 & 0.81 & 0.85 & 0.63 & 5.214 & $<0.001$ & Statistically significant \\
\hline $1^{\text {st }}$ month- $3^{\text {rd }}$ month & 21 & 0.90 & 0.47 & 0.94 & 0.63 & 0.375 & 0.712 & Statistically nonsignificant \\
\hline $1^{\text {st }}$ month- $6^{\text {th }}$ month & 21 & 0.90 & 0.47 & 0.85 & 0.63 & 0.41 & 0.686 & Statistically nonsignificant \\
\hline $3^{\text {rd }}$ month- $6^{\text {th }}$ month & 21 & 0.94 & 0.63 & 0.85 & 0.63 & 2.29 & 0.033 & Statistically nonsignificant \\
\hline
\end{tabular}


Palwankar, et al.: The levels of glutathione, both oxidized and reduced forms in the gingival crevicular fluid

\begin{tabular}{|c|c|c|c|c|c|c|c|c|}
\hline Time interval & Number of subjects & Mean & SD & Mean & SD & $t$ & $P$ & Inference \\
\hline Baseline- $1^{\text {st }}$ month & 20 & 0.23 & 0.15 & 0.19 & 0.26 & 0.654 & 0.521 & Statistically nonsignificant \\
\hline Baseline- $3^{\text {rd }}$ month & 20 & 0.23 & 0.15 & 0.24 & 0.43 & 0.146 & 0.886 & Statistically nonsignificant \\
\hline Baseline- $6^{\text {th }}$ month & 20 & 0.23 & 0.15 & 0.13 & 0.10 & 2.747 & 0.013 & Statistically nonsignificant \\
\hline $1^{\text {st }}$ month- $3^{\text {rd }}$ month & 20 & 0.18 & 0.25 & 0.24 & 0.42 & 0.566 & 0.578 & Statistically nonsignificant \\
\hline $1^{\text {st }}$ month- $6^{\text {th }}$ month & 20 & 0.18 & 0.25 & 0.13 & 0.10 & 0.993 & 0.333 & Statistically nonsignificant \\
\hline $3^{\text {rd }}$ month- $6^{\text {th }}$ month & 20 & 0.24 & 0.42 & 0.13 & 0.10 & 1.173 & 0.255 & Statistically nonsignificant \\
\hline
\end{tabular}

\begin{tabular}{|c|c|c|c|c|c|c|c|c|}
\hline Time interval & Number of subjects & Mean & SD & Mean & SD & $t$ & $P$ & Inference \\
\hline Baseline- $1^{\text {st }}$ month & 20 & 0.20 & 0.09 & 0.21 & 0.27 & 0.235 & 0.817 & Statistically nonsignificant \\
\hline Baseline- $3^{\text {rd }}$ month & 20 & 0.20 & 0.09 & 0.21 & 0.23 & 0.223 & 0.826 & Statistically nonsignificant \\
\hline Baseline $-6^{\text {th }}$ month & 20 & 0.20 & 0.09 & 0.15 & 0.11 & 1.466 & 0.159 & Statistically nonsignificant \\
\hline $1^{\text {st }}$ month- $3^{\text {rd }}$ month & 20 & 0.21 & 0.27 & 0.21 & 0.23 & 0.101 & 0.92 & Statistically nonsignificant \\
\hline $1^{\text {st }}$ month- $6^{\text {th }}$ month & 20 & 0.21 & 0.27 & 0.15 & 0.11 & 1.241 & 0.23 & Statistically nonsignificant \\
\hline $3^{\text {rd }}$ month- $6^{\text {th }}$ month & 20 & 0.21 & 0.23 & 0.15 & 0.11 & 1.375 & 0.185 & Statistically nonsignificant \\
\hline
\end{tabular}

\begin{tabular}{|c|c|c|c|c|c|c|c|c|}
\hline Time interval & Number of subjects & Mean & SD & Mean & SD & $t$ & $P$ & Inference \\
\hline Baseline- $1^{\text {st }}$ month & 20 & 0.16 & 0.08 & 0.19 & 0.23 & 0.36 & 0.722 & Statistically nonsignificant \\
\hline Baseline- $3^{\text {rd }}$ month & 20 & 0.16 & 0.08 & 0.17 & 0.21 & 0.09 & 0.929 & Statistically nonsignificant \\
\hline Baseline- $6^{\text {th }}$ month & 20 & 0.16 & 0.08 & 0.16 & 0.20 & 0.908 & 0.375 & Statistically nonsignificant \\
\hline $1^{\text {st }}$ month- $3^{\text {rd }}$ month & 20 & 0.19 & 0.23 & 0.17 & 0.21 & 0.845 & 0.409 & Statistically nonsignificant \\
\hline $1^{\text {st }}$ month- $6^{\text {th }}$ month & 20 & 0.19 & 0.23 & 0.16 & 0.20 & 0.841 & 0.411 & Statistically nonsignificant \\
\hline $3^{\text {rd }}$ month- $6^{\text {th }}$ month & 20 & 0.17 & 0.21 & 0.16 & 0.20 & 0.882 & 0.389 & Statistically nonsignificant \\
\hline
\end{tabular}

SD: Standard deviation

\begin{tabular}{|c|c|c|c|c|c|c|c|c|}
\hline Time interval & Number of subjects & Mean (Umol/dl) & SD & Mean (Umol/dl) & SD & $t$ & $P$ & Inference \\
\hline Baseline- $1^{\text {st }}$ month & 22 & 0.30 & 0.19 & 1.76 & 0.95 & 7.129 & $<0.001$ & Statistically significant \\
\hline Baseline- $3^{\text {rd }}$ month & 21 & 0.31 & 0.19 & 4.33 & 1.67 & 10.855 & $<0.001$ & Statistically significant \\
\hline Baseline- $6^{\text {th }}$ month & 21 & 0.31 & 0.19 & 5.14 & 1.55 & 14.274 & $<0.001$ & Statistically significant \\
\hline $1^{\text {st }}$ month- $3^{\text {rd }}$ month & 21 & 1.79 & 0.96 & 4.33 & 1.67 & 11.337 & $<0.001$ & Statistically significant \\
\hline $1^{\text {st }}$ month- $6^{\text {th }}$ month & 21 & 1.79 & 0.96 & 5.14 & 1.55 & 9.513 & $<0.001$ & Statistically significant \\
\hline
\end{tabular}

SD: Standard deviation

months, baseline - 6 months, 1-3 months and 1-6 months in Tables 11 and 12.

Ratio of reduced GSH and GSSG for test group at different time interval during the study was reported to be statistically significant $(P<0.001)$ at confidence interval of $95 \%$ at baseline - 1-month, baseline -3 months, baseline -6 months, 1-3 months, 1-6 months [Table 7]. Comparison of the mean ratio of reduced and oxidized GSH (GSH: GSSG) score with a standard deviation between test and control group is depicted in Figure 4. The GSH and GSSG ratio values for control group were shown in Table 13. This improvement in the GSH: GSSG ratio after treatment was as a result of an increase in levels of reduced GSH and a reduction in levels of oxidized GSH.

\section{DISCUSSION}

The present data confirm the study conducted by Chapple et al., 2002 suggesting that a millimolar concentration of GSH was present in GCF, which decreases during periodontitis. The reduction in GSH levels is suggestive of its protective role for vital cells and tissue structures from host-derived free radicals. Due to the conversion of GSH to GSSG, there is a reduction in GSH levels at inflamed tissue sites as 
Palwankar, et al.: The levels of glutathione, both oxidized and reduced forms in the gingival crevicular fluid

\begin{tabular}{|c|c|c|c|c|c|c|}
\hline & Groups & $n$ & Mean & SD & $t$ & $P$ \\
\hline \multirow[t]{2}{*}{ GSH (B) } & Test group & 22 & 108.67 & 42.39 & 15.486 & $<0.001$ \\
\hline & Control group & 20 & 519.34 & 116.29 & & \\
\hline \multirow[t]{2}{*}{ GSSG (B) } & Test group & 22 & 318.41 & 139.69 & 8.538 & $<0.001$ \\
\hline & Control group & 20 & 50.80 & 9.97 & & \\
\hline \multirow[t]{2}{*}{ GSH_GSSG (B) } & Test group & 22 & 0.30 & 0.19 & 15.7 & $<0.001$ \\
\hline & Control group & 20 & 10.73 & 3.11 & & \\
\hline \multirow[t]{2}{*}{ GSH (1-month) } & Test group & 22 & 233.91 & 107.74 & 8.683 & $<0.001$ \\
\hline & Control group & 20 & 525.15 & 109.48 & & \\
\hline \multirow[t]{2}{*}{ GSSG (1-month) } & Test group & 22 & 145.86 & 76.22 & 5.34 & $<0.001$ \\
\hline & Control group & 20 & 53.70 & 12.27 & & \\
\hline \multirow[t]{2}{*}{ GSH_GSSG (1-month) } & Test group & 22 & 1.76 & 0.95 & 13.011 & $<0.001$ \\
\hline & Control group & 20 & 10.31 & 2.92 & & \\
\hline \multirow[t]{2}{*}{ GSH (3 month) } & Test group & 21 & 362.00 & 145.30 & 4.567 & $<0.001$ \\
\hline & Control group & 20 & 533.65 & 86.48 & & \\
\hline \multirow{2}{*}{ GSSG (3 month) } & Test group & 21 & 89.43 & 38.09 & 4.248 & $<0.001$ \\
\hline & Control group & 20 & 51.85 & 10.88 & & \\
\hline \multirow[t]{2}{*}{ GSH_GSSG (3 month) } & Test group & 21 & 4.33 & 1.67 & 9.679 & $<0.001$ \\
\hline & Control group & 20 & 10.70 & 2.48 & & \\
\hline \multirow[t]{2}{*}{ GSH (6 month) } & Test group & 21 & 386.38 & 109.20 & 5.576 & $<0.001$ \\
\hline & Control group & 20 & 555.50 & 82.40 & & \\
\hline \multirow[t]{2}{*}{ GSSG (6 month) } & Test group & 21 & 80.48 & 27.33 & 4.141 & $<0.001$ \\
\hline & Control group & 20 & 52.60 & 12.91 & & \\
\hline \multirow[t]{2}{*}{ GSH_GSSG (6 month) } & Test group & 21 & 5.14 & 1.55 & 8.895 & $<0.001$ \\
\hline & Control group & 20 & 11.08 & 2.61 & & \\
\hline \multirow[t]{2}{*}{ Plaque index (B) } & Test group & 22 & 1.98 & 0.23 & 28.667 & $<0.001$ \\
\hline & Control group & 19 & 0.23 & 0.15 & & \\
\hline \multirow[t]{2}{*}{ SBI (B) } & Test group & 22 & 2.20 & 0.79 & 11.482 & $<0.001$ \\
\hline & Control group & 20 & 0.16 & 0.08 & & \\
\hline \multirow[t]{2}{*}{ Gingival index (B) } & Test group & 22 & 2.23 & 1.31 & 6.938 & $<0.001$ \\
\hline & Control group & 20 & 0.20 & 0.09 & & \\
\hline \multirow[t]{2}{*}{ Plaque index (1-month) } & Test group & 22 & 0.92 & 0.50 & 5.9 & $<0.001$ \\
\hline & Control group & 20 & 0.18 & 0.25 & & \\
\hline \multirow[t]{2}{*}{ SBI (1-month) } & Test group & 22 & 0.93 & 0.48 & 6.336 & $<0.001$ \\
\hline & Control group & 20 & 0.19 & 0.23 & & \\
\hline \multirow[t]{2}{*}{ Gingival index (1-month) } & Test group & 22 & 0.86 & 0.49 & 5.233 & $<0.001$ \\
\hline & Control group & 20 & 0.21 & 0.27 & & \\
\hline \multirow[t]{2}{*}{ Plaque index (3 month) } & Test group & 21 & 0.84 & 0.53 & 3.996 & $<0.001$ \\
\hline & Control group & 20 & 0.24 & 0.42 & & \\
\hline \multirow[t]{2}{*}{ SBI (3 month) } & Test group & 21 & 0.94 & 0.63 & 5.243 & $<0.001$ \\
\hline & Control group & 20 & 0.17 & 0.21 & & \\
\hline \multirow[t]{2}{*}{ Gingival index (3 month) } & Test group & 21 & 0.87 & 0.56 & 4.919 & $<0.001$ \\
\hline & Control group & 20 & 0.21 & 0.23 & & \\
\hline Plaque index (6 month) & Test group & 21 & 0.86 & 0.49 & 6.558 & $<0.001$ \\
\hline & Control group & 20 & 0.13 & 0.10 & & \\
\hline SBI (6 month) & Test group & 21 & 0.85 & 0.63 & 0.803 & 0.427 \\
\hline & Control group & 20 & 0.52 & 1.76 & & \\
\hline Gingival index (6 month) & Test group & 21 & 1.00 & 0.67 & 5.539 & $<0.001$ \\
\hline & Control group & 20 & 0.15 & 0.11 & & \\
\hline
\end{tabular}

also seen in this study. Similarly, the reduced GSH: GSSG ratio at baseline was due to reduced levels of GSH as well as increased accumulation of GSSG. These results were consistent with findings reported in earlier studies demonstrating a reduction in total antioxidant capacity of saliva in patients with chronic periodontitis along with reduced levels of GSH. ${ }^{[15-17]}$

In this study, a significant increase in GSH levels along with improvement in GSH: GSSG ratio at 1-month was 
Palwankar, et al.: The levels of glutathione, both oxidized and reduced forms in the gingival crevicular fluid

\begin{tabular}{|c|c|c|c|c|c|c|c|c|}
\hline Time interval & Number of subjects & Mean (Umol/dl) & SD & Mean (Umol/dl) & SD & $t$ & $\boldsymbol{P}$ & Inference \\
\hline Baseline- $1^{\text {st }}$ month & 22 & 108.67 & 42.39 & 233.91 & 107.74 & 7.64 & $<0.001$ & Statistically significant \\
\hline Baseline- $3^{\text {rd }}$ month & 21 & 112.15 & 40.10 & 362.00 & 145.30 & 9.415 & $<0.001$ & Statistically significant \\
\hline Baseline- $6^{\text {th }}$ month & 21 & 112.15 & 40.10 & 386.38 & 109.20 & 13.262 & $<0.001$ & Statistically significant \\
\hline $1^{\text {st }}$ month- $3^{\text {rd }}$ month & 21 & 240.48 & 105.79 & 362.00 & 145.30 & 8.047 & $<0.001$ & Statistically significant \\
\hline $1^{\text {st }}$ month- $6^{\text {th }}$ month & 21 & 240.48 & 105.79 & 386.38 & 109.20 & 8.746 & $<0.001$ & Statistically significant \\
\hline
\end{tabular}

Table 10: Intragroup comparison of mean oxidized glutathione level and SD in test group

\begin{tabular}{|c|c|c|c|c|c|c|c|c|}
\hline Time interval & Number of subjects & Mean (Umol/dl) & SD & Mean (Umol/dl) & SD & $t$ & $P$ & Inference \\
\hline Baseline- $1^{\text {st }}$ month & 22 & 318.41 & 39.69 & 233.91 & 76.22 & 6.769 & $<0.001$ & Statistically significant \\
\hline Baseline- $3^{\text {rd }}$ month & 21 & 326.76 & 137.39 & 362.00 & 38.09 & 8.755 & $<0.001$ & Statistically significant \\
\hline Baseline- $6^{\text {th }}$ month & 21 & 326.76 & 137.39 & 386.38 & 27.33 & 8.926 & $<0.001$ & Statistically significant \\
\hline $1^{\text {st }}$ month- $3^{\text {rd }}$ month & 21 & 148.52 & 77.04 & 145.86 & 38.09 & 5.119 & $<0.001$ & Statistically significant \\
\hline $1^{\text {st }}$ month- $6^{\text {th }}$ month & 21 & 148.52 & 77.04 & 89.43 & 27.33 & 4.721 & $<0.001$ & Statistically significant \\
\hline $3^{\text {rd }}$ month- $6^{\text {th }}$ month & 21 & 89.43 & 38.09 & 80.48 & 27.33 & 1.565 & 0.133 & Statistically nonsignificant \\
\hline
\end{tabular}

SD: Standard deviation

\begin{tabular}{|c|c|c|c|c|c|c|c|c|}
\hline Time interval & Number of subjects & Mean & SD & Mean & SD & $t$ & $P$ & Inference \\
\hline Baseline- $1^{\text {st }}$ month & 20 & 519.34 & 116.29 & 525.15 & 109.48 & 0.424 & 0.676 & Statistically nonsignificant \\
\hline Baseline- $3^{\text {rd }}$ month & 20 & 519.34 & 116.29 & 533.65 & 86.48 & 0.905 & 0.377 & Statistically nonsignificant \\
\hline Baseline- $6^{\text {th }}$ month & 20 & 519.34 & 116.29 & 555.50 & 82.40 & 1.722 & 0.101 & Statistically nonsignificant \\
\hline $1^{\text {st }}$ month- $3^{\text {rd }}$ month & 20 & 525.15 & 109.48 & 533.65 & 86.48 & 0.602 & 0.554 & Statistically nonsignificant \\
\hline $1^{\text {st }}$ month- $6^{\text {th }}$ month & 20 & 525.15 & 109.48 & 555.50 & 82.40 & 1.834 & 0.082 & Statistically nonsignificant \\
\hline
\end{tabular}

SD: Standard deviation

\begin{tabular}{|c|c|c|c|c|c|c|c|c|}
\hline Time interval & Number of subjects & Mean & SD & Mean & SD & $t$ & $\boldsymbol{P}$ & Inference \\
\hline Baseline- $1^{\text {st }}$ month & 20 & 50.80 & 9.97 & 53.70 & 12.27 & 0.998 & 0.331 & Statistically nonsignificant \\
\hline Baseline- $3^{\text {rd }}$ month & 20 & 50.80 & 9.97 & 51.85 & 10.88 & 0.434 & 0.67 & Statistically nonsignificant \\
\hline Baseline- $6^{\text {th }}$ month & 20 & 50.80 & 9.97 & 52.60 & 12.91 & 0.755 & 0.459 & Statistically nonsignificant \\
\hline $1^{\text {st }}$ month- $3^{\text {rd }}$ month & 20 & 53.70 & 12.27 & 51.85 & 10.88 & 0.96 & 0.349 & Statistically nonsignificant \\
\hline $1^{\text {st }}$ month- $6^{\text {th }}$ month & 20 & 53.70 & 12.27 & 52.60 & 12.91 & 0.641 & 0.529 & Statistically nonsignificant \\
\hline $3^{\text {rd }}$ month- $6^{\text {th }}$ month & 20 & 51.85 & 10.88 & 52.60 & 12.91 & 0.408 & 0.688 & Statistically nonsignificant \\
\hline
\end{tabular}

\begin{tabular}{|c|c|c|c|c|c|c|c|c|}
\hline Time interval & Number of subjects & Mean & SD & Mean & SD & $t$ & $P$ & Inference \\
\hline Baseline- $1^{\text {st }}$ month & 20 & 10.73 & 3.11 & 10.31 & 2.92 & 0.852 & 0.405 & Statistically nonsignificant \\
\hline Baseline- $3^{\text {rd }}$ month & 20 & 10.73 & 3.11 & 10.70 & 2.48 & 0.056 & 0.956 & Statistically nonsignificant \\
\hline Baseline $-6^{\text {th }}$ month & 20 & 10.73 & 3.11 & 11.08 & 2.61 & 0.893 & 0.383 & Statistically nonsignificant \\
\hline $1^{\text {st }}$ month- $3^{\text {rd }}$ month & 20 & 10.31 & 3.11 & 10.70 & 2.48 & 1.181 & 0.252 & Statistically nonsignificant \\
\hline $1^{\text {st }}$ month- $6^{\text {th }}$ month & 20 & 10.31 & 2.92 & 10.70 & 2.61 & 2.192 & 0.041 & Statistically nonsignificant \\
\hline
\end{tabular}

observed following nonsurgical periodontal therapy viz., scaling and root planing, which is consistent with the observations made by several others studies. ${ }^{[18-20]}$ These results were suggestive of an improvement in $\mathrm{AO}$ status and reduction in oxidative stress following nonsurgical periodontal therapy. Other possibilities for the improvement seen following nonsurgical therapy is the reduction in proteolytic activity of microorganisms and also reductions in inflammation posttherapy, thereby lowering levels of oxidants 
Palwankar, et al.: The levels of glutathione, both oxidized and reduced forms in the gingival crevicular fluid

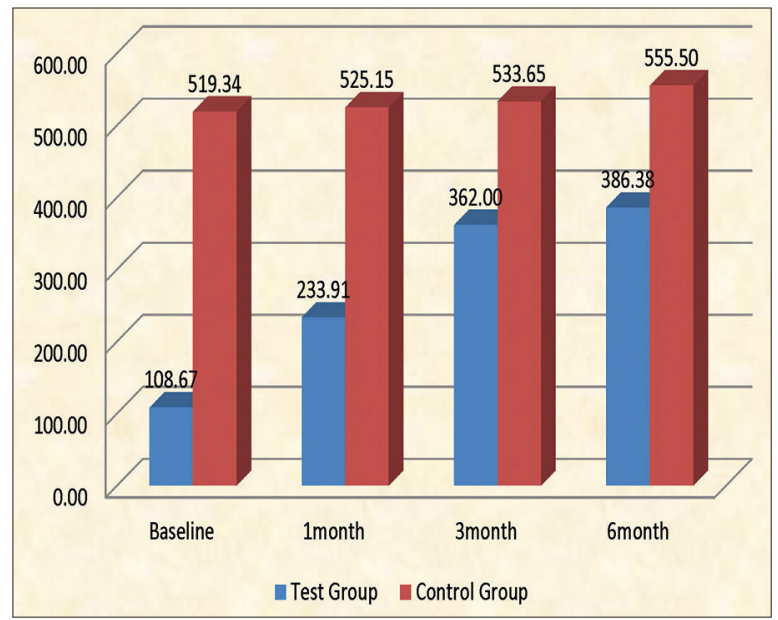

Figure 2: Reduced glutathione levels at different time intervals for test and control groups

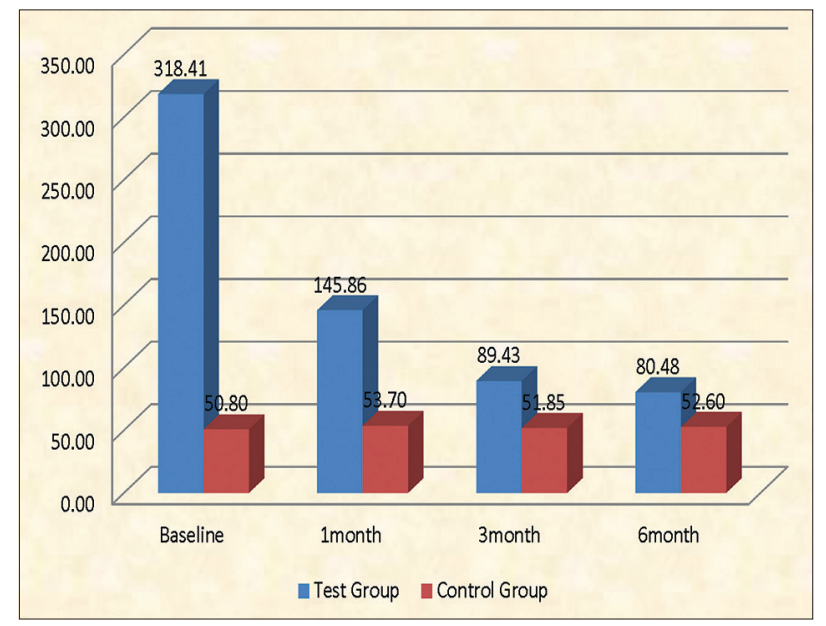

Figure 3: Oxidized glutathione levels at different time intervals for test and control groups

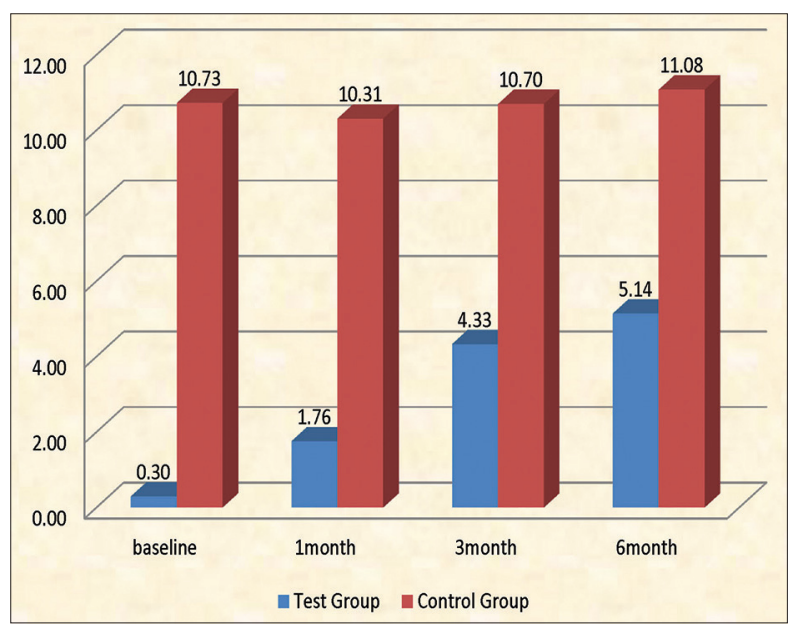

Figure 4: Ratio of reduced to oxidized glutathione levels at different time intervals for test and control groups

in GCF, both exogenously and endogenously. The improvement was seen in GSH: GSSG ratio at
3 months is due to relatively long duration taken by subgingival microflora to reestablish, hence their reduced activity. ${ }^{[2]}$

The preliminary findings of studies stated that millimolar concentrations of GSH is present in GCF gets significantly reduced in chronic periodontitis patients. ${ }^{[14,19]}$ In addition, this study demonstrated that successful, nonsurgical periodontal therapy does restore the redox balance (GSH: GSSG) till 3 months, but at 6 months no significant improvement in either GSH or GSH: GSSG ratio was noted. Thus after 3 months thorough oral hygiene practice is not self-sufficient for restoring the GSH levels and GSH: GSSG ratio to health, this implies that there is a reduced potential of periodontal tissues against ROS activity in chronic periodontitis patients, even after successful nonsurgical therapy. Such findings have opened up the potential of using pharmacological agents to elevate buffering capacity within tissues by elevating GSH levels, e.g. use of the GSH promoting drug $\mathrm{N}$-acetyl cysteine. This pharmacological approach is currently under investigation in the management of rheumatoid arthritis. ${ }^{[20,21]}$ Anti-inflammatory tissue redox state can be created by supplementing the individuals with micronutrients for elevating GSH concentrations. ${ }^{[22]}$ For various chronic inflammatory diseases that are associated with redox imbalance, AO supplementation is actively pursued as a preventive and therapeutic measure.

\section{Limitations}

Duration of the study, the reliability of the spectroscopic method used for GSH estimation and method of GCF collection. This study opens up scope for various future studies dealing with the role of GSH as biological diagnostic disease marker, $\mathrm{AO}$, detoxifier, and immune modulator. Long-term studies can be undertaken to evaluate the efficacy of novel therapeutic approaches for improving the buffering capacity within periodontal tissues by elevating the GSH levels.

\section{CONCLUSIONS}

The study concluded that at baseline due to prevailing oxidative stress the levels of reduced GSH were reduced in patients with chronic periodontitis. Following nonsurgical therapy the levels of GSH were improved at 1 -month and 3 months. At 6 months recall following nonsurgical therapy, an adjunctive use of micronutritional supplements to boost $\mathrm{AO}$ concentration in tissues by preserving GSH or by elevating its level at the inflamed sites is recommended. 


\section{Financial support and sponsorship}

Nil.

\section{Conflicts of interest}

There are no conflicts of interest.

\section{REFERENCES}

1. Socransky SS, Haffajee AD, Cugini MA, Smith C, Kent RL Jr. Microbial complexes in subgingival plaque. J Clin Periodontol 1998;25:134-44.

2. Newman MG, Takei HH, Klokkevoid PR, Carranza FA. Classification of disease and conditions affecting the periodontium. Carranza's Clinical Periodontology. 10 ${ }^{\text {th }}$ ed. St. Louis, Missouri: Saunders; 2009. p. 104.

3. Fredriksson M, Gustafsson A, Asman B, Bergström K. Hyper-reactive peripheral neutrophils in adult periodontitis: Generation of chemiluminescence and intracellular hydrogen peroxide after in vitro priming and FcgammaR-stimulation. J Clin Periodontol 1998;25:394-8.

4. Gustafsson A, Asman B, Bergström K. Priming response to inflammatory mediators in hyperreactive peripheral neutrophils from adult periodontitis. Oral Dis 1997;3:167-71.

5. Loe H, Theilade E, Jensen SB. Experimental gingivitis in man. J Periodontol 1965;36:177-87.

6. Canakci CF, Cicek Y, Yildirim A, Sezer U, Canakci V. Increased levels of 8-hydroxydeoxyguanosine and malondialdehyde and its relationship with antioxidant enzymes in saliva of periodontitis patients. Eur J Dent 2009;3:100-6.

7. Halliwell B. Reactive oxygen species in living systems: Source, biochemistry, and role in human disease. Am J Med 1991;91:14S-22S.

8. Battino $M$, Bullon $\mathrm{P}$, Wilson $\mathrm{M}$, Newman $\mathrm{H}$. Oxidative injury and inflammatory periodontal diseases: The challenge of anti-oxidants to free radicals and reactive oxygen species. Crit Rev Oral Biol Med 1999:10:458-76.

9. Sies H. Oxidative stress, angewandte chemie int . London academic press $1986 ; 25: 1058-71$.

10. Cnubben NH, Rietjens IM, Wortelboer H, van Zanden J, van Bladeren PJ. The interplay of glutathione-related processes in antioxidant defense. Environ Toxicol Pharmacol 2001;10:141-52.

11. Townsend DM, Tew KD, Tapiero H. The importance of glutathione in human disease. Biomed Pharmacother 2003;57:145-55.
12. Meister A, Anderson ME. Glutathione. Annu Rev Biochem 1983;52:711-60.

13. Beutler E, Duron O, Kelly BM. Improved method for the determination of blood glutathione. J Lab Clin Med 1963;61:882-8.

14. Chapple IL, Brock G, Eftimiadi C, Matthews JB. Glutathione in gingival crevicular fluid and its relationship to local antioxidant capacity in periodontal health and disease. J Clin Periodontol 2002;42:1-73.

15. Borges I Jr, Moreria EA, Filho DW, de Oliveira TB, Da Silva MB, Frode TS. Proinflammatory and oxidative stress markers in patients with periodontal disease. Mediators Inflamm 2007;12:457-64.

16. Tsai CC, Chen HS, Ho YP, Ho KY, Wu YM, Hou GL. Periodontopathogens and Oxidative Stress in Periodontal Diseases. Vol. 6. Paper 0595 $81^{\text {st }}$ General Session of International Association for Dental Research; 2003. p. 25-8

17. Moore S, Calder KA, Miller NJ, Rice-Evans CA. Antioxidant activity of saliva and periodontal disease. Free Radic Res 1994;21:417-25.

18. Guentsch A, Preshaw PM, Bremer-Streck S, Klinger G, Glockmann E, Sigusch BW. Lipid peroxidation and antioxidant activity in saliva of periodontitis patients: Effect of smoking and periodontal treatment. Clin Oral Investig 2008;12:345-52

19. Grant MM, Brock GR, Matthews JB, Chapple IL. Crevicular fluid glutathione levels in periodontitis and the effect of non-surgical therapy. J Clin Periodontol 2010;37:17-23.

20. Reiners JJ Jr, Mathieu P, Okafor C, Putt DA, Lash LH. Depletion of cellular glutathione by conditions used for the passaging of adherent cultured cells. Toxicol Lett 2000;115:153-63.

21. Kelly GS. Clinical applications of N-acetylcysteine. Altern Med Rev 1998;3:114-27.

22. Johnston CS, Meyer CG, Srilakshmi JC. Vitamin C elevates red blood cell glutathione in healthy adults. Am J Clin Nutr 1993;58:103-5.

\begin{tabular}{|l|l|}
\hline \multicolumn{3}{|c|}{ Access this article online } \\
\hline Quick Response Code: & \\
\hline
\end{tabular}

